\title{
Insecticide resistance in Anopheles stephensi in Somali Region, eastern Ethiopia
}

\author{
Solomon Yared ${ }^{1 *} \mathbb{0}$, Araya Gebressielasie ${ }^{2}$, Lambodhar Damodaran ${ }^{3}$, Victoria Bonnell ${ }^{4}$, Karen Lopez ${ }^{5}$, \\ Daniel Janies ${ }^{5}$ and Tamar E. Carter ${ }^{6}$
}

\begin{abstract}
Background: The movement of malaria vectors into new areas is a growing concern in the efforts to control malaria. The recent report of Anopheles stephensi in eastern Ethiopia has raised the necessity to understand the insecticide resistance status of the vector in the region to better inform vector-based interventions. The aim of this study was to evaluate insecticide resistance in An. stephensi in eastern Ethiopia using two approaches: (1) World Health Organization (WHO) bioassay tests in An. stephensi; and (2) genetic analysis of insecticide resistance genes in An. stephensi in eastern Ethiopia.

Methods: Mosquito larvae and pupae were collected from Kebri Dehar. Insecticide susceptibility of An. stephensi was tested with malathion $5 \%$, bendiocarb $0.1 \%$, propoxur $0.1 \%$, deltamethrin $0.05 \%$, permethrin $0.75 \%$, pirimiphosmethyl $0.25 \%$ and DDT $4 \%$, according to WHO standard protocols. In this study, the knockdown resistance locus (kdr) in the voltage gated sodium channel (vgsc) and ace $R$ locus in the acetylcholinesterase gene (ace-1) were analysed in An. stephensi.
\end{abstract}

Results: All An. stephensi samples were resistant to carbamates, with mortality rates of 23\% and 21\% for bendiocarb and propoxur, respectively. Adult An. stephensi was also resistant to pyrethroid insecticides with mortality rates $67 \%$ for deltamethrin and 53\% for permethrin. Resistance to DDT and malathion was detected in An. stephensi with mortality rates of $32 \%$ as well as An. stephensi was resistance to pirimiphos-methyl with mortality rates $14 \%$. Analysis of the insecticide resistance loci revealed the absence of $k d r$ L1014F and L1014S mutations and the ace $1 R$ G119S mutation.

Conclusion: Overall, these findings support that An. stephensi is resistant to several classes of insecticides, most notably pyrethroids. However, the absence of the $k d r$ L1014 gene may suggest non-target site resistance mechanisms. Continuous insecticide resistance monitoring should be carried out in the region to confirm the documented resistance and exploring mechanisms conferring resistance in An. stephensi in Ethiopia.

Keywords: Malaria, Anopheles stephensi, Insecticide resistance, Kebri Dehar, Somali Region

\section{Background}

Malaria remains a major health problem in 2017, an estimated 219 million cases of malaria occurred worldwide, with 435,000 deaths [1]. In Ethiopia, malaria remains a major public health concern with millions of cases and thousands of deaths reported annually [2]. Unlike most

\footnotetext{
*Correspondence: solyar2005@yahoo.com

1 Department of Biology, Jigjiga University, Jigjiga, Ethiopia

Full list of author information is available at the end of the article
}

of the African continent, malaria can be caused by infection with Plasmodium vivax or Plasmodium falciparum. Efforts to control the transmission of malaria currently target Anopheles arabiensis, the primary malaria vector in Ethiopia, as well the secondary vectors Anopheles funestus, Anopheles pharoensis, and Anopheles nili [3]. Successes in reducing the malaria burden could be threatened by the recent detection of the South Asian urban vector Anopheles stephensi in the Horn of Africa, as its role in malaria transmission in Ethiopia is not yet

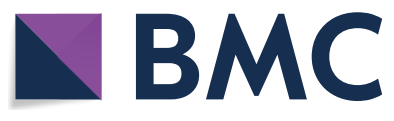

(c) The Author(s) 2020. This article is licensed under a Creative Commons Attribution 4.0 International License, which permits use, sharing, adaptation, distribution and reproduction in any medium or format, as long as you give appropriate credit to the original author(s) and the source, provide a link to the Creative Commons licence, and indicate if changes were made. The images or other third party material in this article are included in the article's Creative Commons licence, unless indicated otherwise in a credit line to the material. If material is not included in the article's Creative Commons licence and your intended use is not permitted by statutory regulation or exceeds the permitted use, you will need to obtain permission directly from the copyright holder. To view a copy of this licence, visit http://creativeco mmons.org/licenses/by/4.0/. The Creative Commons Public Domain Dedication waiver (http://creativecommons.org/publicdomain/ zero/1.0/) applies to the data made available in this article, unless otherwise stated in a credit line to the data. 
confirmed. This mosquito was first detected in the Somali Regional State of Ethiopia in 2016 [4] and has subsequently been confirmed to have a broad distribution in Northeast and east Ethiopia [5]. Anopheles stephensi was also been reported in Djibouti in 2014 [6] and there are now concerns that this species may spread throughout the African continent [7].

In the past decade, Ethiopia has made significant progresses in expanding coverage of key malaria interventions throughout the country. Indoor residual spraying (IRS) and long-lasting insecticidal nets (LLINs) are used in malaria prevention and control strategy in Ethiopia [8]. IRS was first introduced in Ethiopia in 1959 and continues to use as main malaria intervention method. LLINs distributed throughout Ethiopia particularly in Somali Region, about 80 percent of existing LLINs in households were used the night before and the proportion of LLINs used in malarious areas [9]. In Somali region carbamate insecticides have been frequently sprayed during active malaria season in collaboration by Federal Ministry of Health (FMOH) with the regional Health Bureau.

One major obstacle to vector control in Ethiopia and elsewhere is the ever-developing insecticide resistance as a result of indiscriminate and rampant use of the synthetic chemicals in public health and agriculture [10-12]. Pyrethroids remain the only class of insecticides recommended for the treatment of LLINs and accounted for a large proportion of the insecticide used for IRS in Ethiopia and elsewhere in Africa $[8,13]$. This heavy reliance on a single insecticide class has caused mosquito species to develop insecticide resistance. In mosquitoes, pyrethroid resistance is mainly attributed to two major mechanisms: target-site insensitivity and metabolic-based resistance. Target-site resistance is due to mutations in the voltagegated sodium channel on the mosquito's neurons that prevent the insecticide's ability to interfere with the closing of sodium channel that would usually result in paralysis (knockdown) [14]. The knockdown $(k d r)$ mutation L1014 has been observed across multiple Culicidae. Metabolic resistance mediated by detoxifying enzymes also plays a significant role in insecticide resistance in malaria vectors $[15,16]$. The over-expression of detoxification enzymes such as cytochrome $\mathrm{P} 450$ monooxygenases (P450s), carboxylcholinesterases (CCEs) and glutathione $\mathrm{S}$-transferases (GSTs) in mosquitoes are frequently associated with resistance to different classes of insecticides [17].

Insecticide resistance in An. stephensi has been reported in Afghanistan, Pakistan, Dubai, and India [18-21]. In these regions, the frequency of the $k d r$ L1014 mutation varies with strong support for metabolic resistance as well as target site resistance playing a role in An. stephensi. In Ethiopia, studies on An. arabiensis in the western portion of the country report phenotypic resistance to pyrethroids along with L1014 variant [22]. In addition, in Southwest Ethiopia, pre-exposure of $A n$. arabiensis to piperonyl butoxide (PBO) significantly increased vector susceptibility to deltamethrin and permethrin, suggesting both metabolic and target-site mutation mechanisms are responsible for insecticide resistance [23]. Data on the insecticide resistance status of malaria vectors in the eastern portion of the country is lacking, including that of the recently identified $A n$. stephensi. Knowing the status of insecticide resistance of local malaria vectors can aid with vector control planning that involves the use of insecticides. Here the aim of this study was to determine the insecticide susceptibility status of east Ethiopian An. stephensi using bioassay tests and characterizing resistance mechanisms using molecular analysis.

\section{Methods}

\section{Study area}

Our samples were collected in Kebri Dehar, a small town in the Somali Regional State, as previously detailed [4]. This town has a tropical semiarid climate with typically bimodal rainfall patterns. The area is also known to experience recurrent droughts. The population size is over 100,000 individuals, many of whom are pastoralists.

\section{Larval sampling and rearing procedure}

Larvae samples were collected from Kebri Dehar small town in Somali Region in eastern Ethiopia as previously detailed (Fig. 1) [4]. This region is predominantly lowland plain, with sparse vegetation including trees and shrubby. Larvae and pupae of Anopheles were collected from likely larval breeding habitats including man-made water containers, fresh water pools, stream margins, discarded tires, plastic containers, cisterns, barrels. In the study area water is stored in a container locally called "Birka" and it is constructed from cement and stone, the local people used for drinking and construction purposes. Briefly, immature stages of An. stephensi were collected from breeding sites in Kebri Dehar town using the dipping method according to WHO guidelines and were reared to adulthood in the field laboratory (Fig. 2). Collections took place from November to December 2016. In the field laboratory, the larvae and pupae were maintained at $28 \pm 2{ }^{\circ} \mathrm{C}$ and $70 \pm 10 \%$ relative humidity. The pupae were sorted and transferred with pipettes from the enamel trays to beakers with small amounts of water. Each beaker was placed inside a cage and was provided with $10 \%$ sugar solution for rearing them in the cage (Fig. 2). After two to three days, the pupae emerged to adults and the cages were put in safe place protected from contamination, ants, and other 


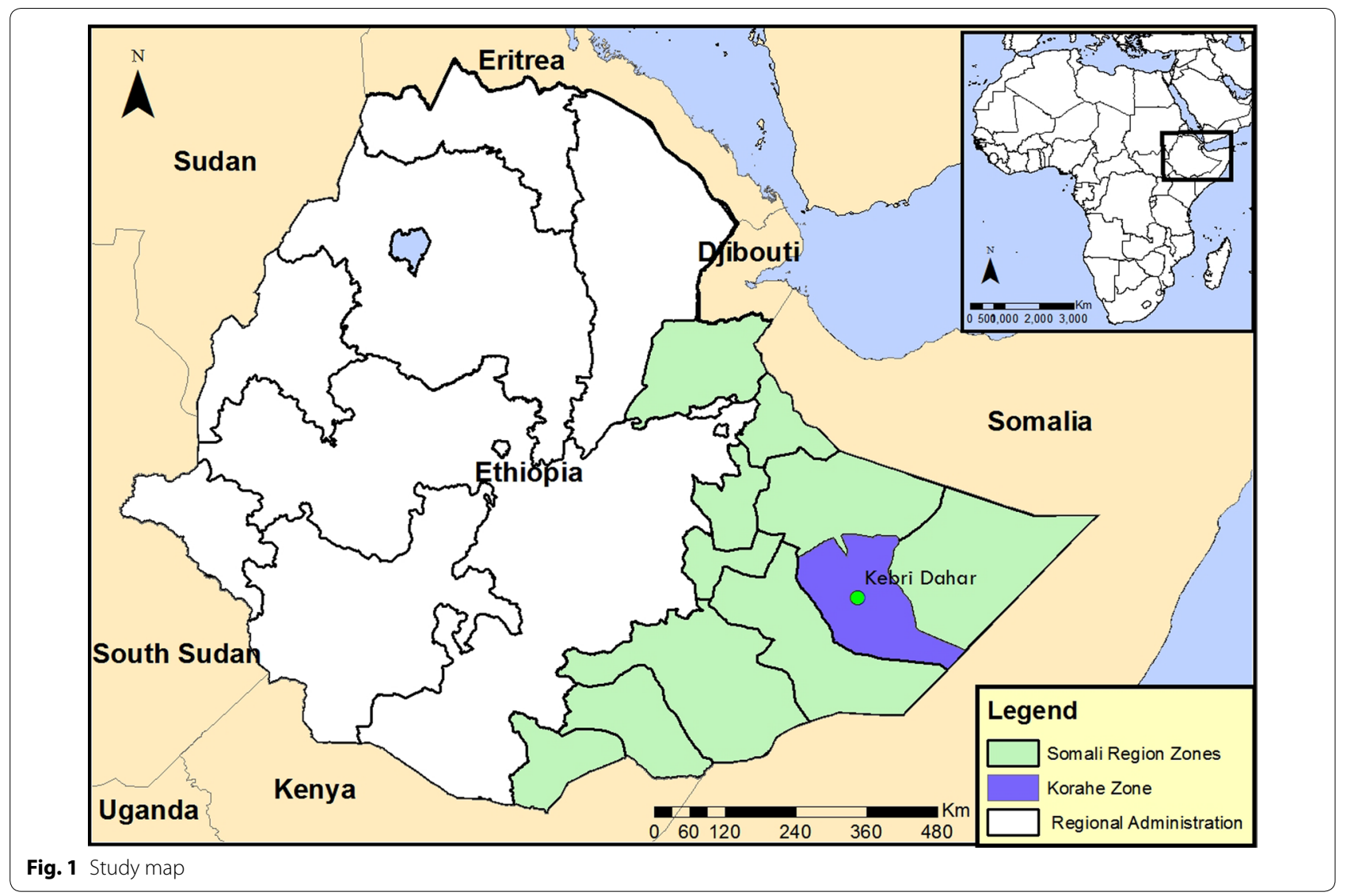

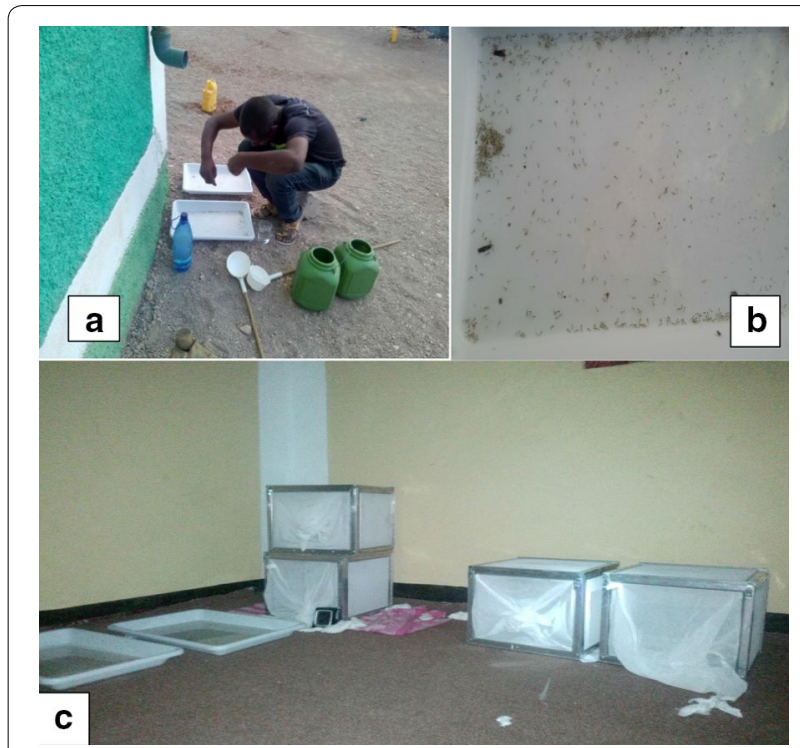

Fig. 2 Rearing larvae to adult at field laboratory: a Feeding yeast to larvae, $\mathbf{b}$ larvae and pupae, c emerging adult in side cages

insects. The laboratory reared females of An. stephensi were used for different insecticide susceptibility test using WHO bioassay.

\section{Mosquito identification}

Species identification of the mosquitoes was conducted using standard morphological keys [24, 25] and molecular analysis of ITS2 and COI loci as reported previously for An. stephensi [4].

\section{Insecticide susceptibility test for Anopheles stephensi}

Insecticide susceptibility tests were carried out following WHO insecticide susceptibility test procedure [26]. A total of 700 non-blood fed adult female An. stephensi (2-3 day-old) were exposed to insecticide impregnated papers with discriminating concentrations of DDT (4\%), Pirimiphos-methyl 0.25\%, malathion (5\%), deltamethrin $(0.05 \%)$, permethrin $0.75 \%$, bendiocarb $(0.1 \%)$, and propoxur $(0.1 \%)$. Batches of 25 mosquitoes in four replicates were exposed to insecticide impregnated papers for $1 \mathrm{~h}$ in WHO test tubes. The knockdown effects for all tested insecticides were recorded at 10,15, 20,30,40,50, and $60 \mathrm{~min}$. A control in two replicates (50 female An. stephensi were used for each insecticide), each with equal number of mosquitoes, exposed to papers impregnated with oil was run concurrently. Mosquitoes were then transferred into holding tubes with untreated papers, where they were supplied with $10 \%$ sucrose solution. 
And, mortality was recorded 24-h post exposure. When mosquito mortality rate in the control is between 5 and $20 \%$, mortality was corrected using Abbott's formula [27]. All survived and dead specimens following bioassay were kept individually in Eppendorf tubes in silica gel for further molecular identification and $k d r$ PCR assays. Determination of resistance was based on WHO criteria as follows: $98-100 \%$ mortality indicates susceptibility, 90 97\% mortality indicates resistance candidate (more investigation is needed) and less than $90 \%$ mortality suggests resistance [26].

Amplification and sequencing of insecticide resistance loci In order to evaluate the presence of insecticide resistance mutations in the An. stephensi collected, a portion of An. stephensi from each experimental arm were selected for genotyping. For kdr mutation analysis, PCR was used to amplify the region of the $v g s c$ gene that housed the L1014 alleles. Mosquito legs were used as templates for DNA. Mosquitoes were PCR amplified and sequenced individually. Analysis of the vgsc was completed according to Singh et al. [20]. PCR amplification of a portion the acetylcholinesterase gene (ace-1), associated with resistance to organophosphates and carbamates [28] was also performed for An. stephensi according to the protocol detailed in [29]. Vgsc and ace-1 PCR reactions were performed at 25 $\mu \mathrm{l}$ total with 2X Promega Hot Start Master Mix (Promega, Madison, W), with $1 \mu \mathrm{l}$ template DNA, and the primer conditions listed in Table 1. Temperature protocols for $v s g c$ amplification were as follows 95 for $5 \mathrm{~min}, 35$ cycles of 95 for $30 \mathrm{~s}, 50$ for $30 \mathrm{~s}, 72$ for $45 \mathrm{~s}, 72$ for $7 \mathrm{~min}$. Temperature protocols for ace- 1 were as follows: 94 for $5 \mathrm{~min}, 35$ cycles of 94 for $30 \mathrm{~s}, 54$ for $30 \mathrm{~s}$, and 72 for $30 \mathrm{~s}, 72$ for $5 \mathrm{~min}$. PCR products were run on $2 \%$ agarose gel for $1 \mathrm{~h}$ at $100 \mathrm{~V}$ to confirm successful PCR amplification.

\section{Analysis of $v g s c$ and ace- 1 sequences}

Sequences were submitted as queries to the National Center for Biotechnology Information's (NCBI) Basic
Local Alignment Search Tool (BLAST) to confirm correct loci were amplified. Sequences were then aligned to identify $k d r$ L1014 and ace-1R G1109 mutations. The allele and genotype frequencies of these mutations were then calculated.

\section{Results \\ Anopheles stephensi insecticide resistance}

A total of 1200 An. stephensi larvae and pupae were collected from the breeding sites. Anopheles stephensi larvae occurred more frequently in cemented water reservoir and plastic water reservoir for construction. Anopheles stephensi positive habitats were mainly located close to human dwelling. Other Larvae and pupae of Aedes and Culex mosquitoes were visually detected and coexisted with An. stephensi, but not recorded.

\section{Bioassay results}

A total of 700 An. stephensi were tested with different insecticides based on WHO protocol. The results of the susceptibility status of populations of An. stephensi are presented in Table 2. Overall, the percent mortality after exposure to insecticides ranged from 14\% (pirimiphos-methyl) to 67\% (deltamethrin). Using the WHO mortality threshold of above $98 \%$, An. stephensi demonstrated resistance to bendiocarb, propoxur, DDT, malathion and permethrin.

\section{Anopheles stephensi insecticide resistance mutations}

A total of 51 mosquitoes were selected randomly from each research arm to represent the natural population of An. stephensi, including 19 that were tested for resistance to deltamethrin, permethrin, or DDT. Of these, eight were resistant to one of these insecticides. Of the $51 A n$. stephensi examined for $k d r$ mutations, none carried the L1014 mutation. In addition, $30 \mathrm{An}$. stephensi were analysed for ace 1 mutations. Of these, 20 had been tested for resistance to bendiocarb, propoxur, or malathion and eight were found to be resistant. Overall, none of the $A n$. stephensi genotyped carried the ace1R G119S mutation.

Table 1 List of primer and conditions used for PCR amplification of portions of the voltage gated sodium channel and acetylcholinesterase genes

\begin{tabular}{|c|c|c|c|c|}
\hline Assay & Primer & Sequence & Annealing temperature $\left({ }^{\circ} \mathrm{C}\right)$ & $\begin{array}{l}\text { Final } \\
\text { concentration } \\
(\mu \mathrm{M})\end{array}$ \\
\hline \multicolumn{5}{|c|}{ Voltage gated sodium channel } \\
\hline & $\mathrm{KdrF}$ & GGACCAYGATTTGCCAAGAT & 50 & 1.25 \\
\hline & VGS_1R & CGAAATTGGACAAAAGCAAGG & 50 & 1.25 \\
\hline \multicolumn{5}{|c|}{ Acetylcholinesterase } \\
\hline & Ex3AGdir & GATCGTGGACACCGTGTTCG & 56 & 1 \\
\hline & Ex3AGrev & AGGATGGCCCGCTGGAACAG & 56 & 1 \\
\hline
\end{tabular}


Table 2 Percentage of mortality of Anopheles stephensi in different insecticide in Kebri Dehar town

\begin{tabular}{lllll}
\hline $\begin{array}{l}\text { Insecticides discriminating } \\
\text { concentration (\%) }\end{array}$ & Classification & No. of An. stephensi tested & $\begin{array}{l}\text { Mortality rate after 24 h } \\
(\%)\end{array}$ & $\begin{array}{l}\text { Resistance? } \\
(<98 \%)\end{array}$ \\
\hline Bendiocarb 0.1\% & Carbamates & 100 & 23 & Yes \\
Propoxure 0.1\% & Carbamates & 100 & 21 & Yes \\
Deltamethrin 0.05\% & Pyrethroid & 100 & 67 & Yes \\
Permethrin 0.75\% & Pyrethroid & 100 & 53 & Yes \\
Malathion 5\% & Organophosphates & 100 & 32 & Yes \\
DDT 4\% & Organochlorine & 100 & 32 & Yes \\
Pirimiphos-methyl 0.25\% & Organophosphates & 100 & 14 & Yes \\
\hline
\end{tabular}

\section{Discussion}

This is the first report of An. stephensi in Ethiopia exhibiting insecticide resistance. What is most concerning is that An. stephensi showed resistance to seven insecticides included in this study highlighting a potential challenge with insecticide-based vector control in this region. This could be An. stephensi is quickly adapting and invading new environment, even survives extremely high temperatures during the dry season [7]. There is some consistency with previous studies on An. stephensi resistance to insecticides. As in the present study, An. stephensi was shown to be resistant to DDT in Iran [30] (Gorouhi et al. 2016). Similarly, a study on An. stephensi in Afghanistan revealed resistance to deltamethrin, malathion, permethrin and DDT [31]. In addition, An. stephensi carbamate resistance was observed in a recent study in Iran [32] as observed in the present study. However, there were some difference between our findings on An. stephensi and previous reports, where An. stephensi was found to be susceptible to the pyrethroids (deltamethrin and permethrin) and malathion in Iran [30, 33] and Pakistan [34]. These differences may reflect differences in the type and extent of insecticide use in Ethiopia compared to other countries.

One surprising finding from our study was the absence of $k d r$ mutation with phenotypic evidence of pyrethroid resistance. The absence of $k d r$ mutations in pyrethroid resistant Anopheles is rare but not unprecedented. A study conducted on An. stephensi collected in Afghanistan revealed a low frequency of L1014 wild-type mutation (44\%) in mosquitoes and a lack of homozygotes of the mosquitoes that were resistant to deltamethrin [35]. The phenotypic presentation of resistance in the majority of An. stephensi specimens with the absence or low frequency of $k d r$ mutations may suggest that metabolic resistance as opposed to targeted resistance is the primary resistance mechanisms in the Ethiopian An. stephensi. A follow-up study on cytochrome P450s, esterases, glutathione S-transferases (GSTs) and acetylcholine esterase (AChE) activities in pyrethroid resistant mosquitoes in Afghanistan further highlight the role of metabolic resistance in An. stephensi [21]. It is also possible the other variants in the $v g s c$ gene may lead to resistance. Additional sequencing and analysis of the entire vgsc should be conducted for identification of other mutations that could lead insecticide resistance.

There are some similarities between these findings on An. stephensi resistance and what has been reported in An. arabiensis in Ethiopia. In An. arabiensis, resistance has been reported for insecticides belonging to all four chemical classes approved for IRS and LLINs. These include DDT (organochlorine), malathion (organophosphate), bendiocarb and propoxur (carbamates) and alpha-cypermethrin, cyfluthrin, deltamethrin, etofenprox, lambda-cyhalothrin and permethrin (pyrethroids) [36-40]. However, the frequencies of $k d r$ mutations are much higher in the Ethiopian An. arabiensis $[36,40]$ than what is reported here. These findings suggest that while, insecticides may induce the development of resistance over time, mechanisms for resistance may vary across species. In depth cross-species genetic analysis for selective signatures on the $v g s c$ and unidentified loci across the genome are needed and underway to further elucidate the differing mechanisms for insecticide resistance in Anopheles species.

In the present study, no ace-1 mutations were observed. There is still some ambiguity around the significance of the ace-1 mutation has been proposed to induce resistance to organophosphates and carbamates resistance [28]. The mutation was absent in the An. stephensi tested in the present study this may reflect the history of the type of insecticides used in the region.

\section{Conclusion}

The finding of multi-insecticide resistance in An. stephensi in Kebri Dehar, Ethiopia emphasizes the need for additional investigation in other parts of Ethiopia and the Horn of Africa. These findings are of importance in the planning and implementation of malaria vector control 
strategy in the region. Additionally, only a portion of the available insecticides were tested in this study. Resistance investigations should include some of the other compound classes including lambda-cyhalorthrin, temephos and chlorpyriphos. The complete absence of the mutation is unexpected but may be due to the An. stephensi collection being limited to one site. Future studies should evaluate the frequency of this mutation in other regions of Ethiopia. The Federal Ministry of Health of Ethiopia should implement appropriate resistance management strategies and integrated vector control intervention.

\begin{abstract}
Abbreviations
ace-1: acetylcholinesterase gene; BLAST: Basic Local Alignment Search Tool; cox1: cytochrome c oxidase subunit 1; CCEs: carboxylcholinesterases; DNA: deoxyribonucleic acid; FMOH: Federal Minster of Health; GSTs: glutathione S-transferases; ITS2: internal transcribed spacer 2; IRS: Indoor Residual Spraying; LLINs: Long Lasting Insecticidal Nets; kdr: knockdown resistance; NCBI: National Center for Biotechnology Information; PCR: polymerase chain reaction; RNA: ribonucleic acid; SRS: Somali Regional Sate; vgsc: voltage gated sodium channel; WHO: World Health Organization.
\end{abstract}

\section{Acknowledgements}

Our gratitude goes to Jigjiga University for the financial support. It is our great pleasure to thank Mr. Geleta Bekele for his technical support in rearing mosquito at field laboratory.

\section{Authors' contributions}

SY: conceptualizing the study, data analysis, and manuscript preparation, reared mosquitoes, tested bioassay; AG: reared mosquitoes, tested bioassay and manuscript preparation; TC: helped with the molecular analysis, design of the molecular analysis, writing the manuscript LD, VB, KL: helped with the molecular analysis DJ: provide resources and facilitated the molecular analysis. All authors read and approved the final manuscript.

\section{Funding}

This study was financially supported by Jigjiga University. This project was partially funded by the University of North Carolina at Charlotte Multicultural Postdoctoral Fellowship.

\section{Availability of data and materials}

The datasets analysed in this study are available from the corresponding author on reasonable request.

Ethics approval and consent to participate

Not applicable.

\section{Consent for publication}

Not applicable.

\section{Competing interests}

The authors declare that they have no competing interests.

\section{Author details}

${ }^{1}$ Department of Biology, Jigjiga University, Jigjiga, Ethiopia. ${ }^{2}$ Department of Zoological Sciences, Addis Ababa University, Addis Ababa, Ethiopia. ${ }^{3}$ Institute of Bioinformatics, University of Georgia, Athens, GA, USA. ${ }^{4}$ Department of Molecular Biology and Biochemistry, Pennsylvania State University, State College, PA, USA. ${ }^{5}$ Department of Bioinformatics and Genomics, University of North Carolina at Charlotte, Charlotte, NC, USA. ${ }^{6}$ Department of Biology, Baylor University, Waco, TX, USA.

Received: 27 November 2019 Accepted: 4 May 2020

Published online: 12 May 2020
References

1. WHO. World malariar 2018. Geneva: World Health Organization; 2018.

2. WHO. World malaria report 2017. Geneva: World Health Organization; 2017.

3. Ghebreyesus A, Deressa W, Witten K, Getachew A, Seboxa T. Malaria. In: Berhane Y, Haile Marim D, Kloos H, editors. Epidemiology and ecology of health and disease in Ethiopia. Addis Ababa: Shama Books; 2006. p. $556-76$.

4. Carter TE, Yared S, Gebresilassie A, Bonnell V, Damodaran L, Lopez K, et al. First detection of Anopheles stephensi Liston, 1901 (Diptera: Culicidae) in Ethiopia using molecular and morphological approaches. Acta Trop. 2018;188:180-6.

5. Balkew M, Mumba P, Dengela D, Yohannes G, Getachew D, Yared S, et al. Geographical distribution of Anopheles stephensi in eastern Ethiopia. Parasit Vectors. 2020;13:35.

6. Seyfarth M, Khaireh BA, Abdi AA, Bouh SM, Faulde MK. Five years following first detection of Anopheles stephensi (Diptera: Culicidae) in Djibouti, Horn of Africa: populations established-malaria emerging. Parasitol Res. 2019;1 18:725-32.

7. WHO. Vector alert: Anopheles stephensi invasion and spread: Horn of Africa, the Republic of the Sudan and surrounding geographical areas, and Sri Lanka. Geneva: World Health Organization; 2019.

8. Kenea O, Balkew M, Tekie H, Deressa W, Loha E, Lindtjørn B, et al. Impact of combining indoor residual spraying and long-lasting insecticidal nets on Anopheles arabiensis in Ethiopia: results from a cluster randomized controlled trial. Malar J. 2019;18:182.

9. Institute Ethiopian Public Health. Ethiopia National Malaria Indicator Survey 2015. Ethiopia: Addis Ababa; 2016.

10. Fettene M, Olana D, Christian RN, Koekemoer LL, Coetzee M. Insecticide resistance in Anopheles arabiensis from Ethiopia. Afr Entomol. 2013;21:89-94.

11. Reid MC, MCKenzie FE. The contribution of agricultural insecticide use to increasing insecticide resistance in African malaria vectors. Malar J. 2016;15:107.

12. Rivero A, Vézilier J, Weill M, Read AF, Gandon S. Insecticide control of vector-borne diseases: when is insecticide resistance a problem? PLoS Pathog. 2010;6:e1001000.

13. WHO. Indoor residual spraying: an operational manual for indoor residual spraying (IRS) for malaria transmission control and elimination. 2nd ed. Geneva: World Health Organization; 2015.

14. Ranson H, Jensen B, Vulule JM, Wang X, Hemingway J, Collins FH. Identification of point mutation in the voltage-gated sodium channel gene of Kenyan Anopheles gambiae associated with resistance to DDT and pyrethroids. Insect Mol Biol. 2000;9:491-7.

15. Hemingway J, Ranson H. Insecticide resistance in insect vectors of human disease. Annu Rev Entomol. 2000;45:371-91.

16. Liu N. Insecticide resistance in mosquitoes: impact, mechanisms, and research directions. Annu Rev Entomol. 2015;60:537-59.

17. David JP, Ismail HM, Chandor-Proust A, Paine MJ. Role of cytochrome P450s in insecticide resistance: impact on the control of mosquito-borne diseases and use of insecticides on Earth. Philos Trans R Soc Lond B Biol Sci. 2013;368:20120429.

18. Hemingway J. The biochemical nature of malathion resistance in Anopheles stephensi from Pakistan. Pestic Biochem Physiol. 1982;17:149-55.

19. Enayati $A A$, Vatandoost $H$, Ladonni $H$, Townson $H$, Hemingway J. Molecular evidence for a kdr-like pyrethroid resistance mechanism in the malaria vector mosquito Anopheles stephensi. Med Vet Entomol. 2003;17:138-44.

20. Singh OP, Dykes $C L$, Lather $M$, Agrawal OP, Adak T. Knockdown resistance (kdr)-like mutations in the voltage-gated sodium channel of a malaria vector Anopheles stephensi and PCR assays for their detection. Malar J. 2011;10:59.

21. Safi NH, Ahmadi AA, Nahzat S, Ziapour SP, Nikookar SH, Fazeli-Dinan M, Enayati A, Hemingway J. Evidence of metabolic mechanisms playing a role in multiple insecticides resistance in Anopheles stephensi populations from Afghanistan. Malar J. 2017;16:100.

22. Alemayehu E, Asale A, Eba K, Getahun K, Tushune K, Bryon A, et al. Mapping insecticide resistance and characterization of resistance mechanisms in Anopheles arabiensis (Diptera: Culicidae) in Ethiopia. Parasit Vectors. 2017;10:407.

23. Yewhalaw D, Asale A, Tushune K, Getachew Y, Duchateau L, Speybroeck N. Bio-efficacy of selected long-lasting insecticidal nets against 
pyrethroid resistant Anopheles arabiensis from South-Western Ethiopia. Parasit Vectors. 2012;5:159.

24. Gillies MT, Coetzee M. A supplement to the Anophelinae of Africa South of the Sahara (Afrotropical Region). Publ S Afr Inst Med Res. 1987:55:1-143.

25. Glick JI. Illustrated key to the female Anopheles of Southwestern Asia and Egypt (Diptera: Culicidae). Mosq Syst. 1992;24:125-53.

26. WHO. Test procedures for insecticide resistance monitoring in malaria vector mosquitoes. Geneva: World Health Organization; 2013. http:// www.who.int/malaria/publications/atoz/9789241505154/en/. Accessed 12 Sept 2016).

27. Abbot WS. A method of computing the effectiveness of an insecticide. J Econ Entomol. 1925;18:265-7.

28. Weill M, Lutfalla G, Mogensen K, Chandre F, Berthomieu A, Berticat C, et al. Comparative genomics: insecticide resistance in mosquito vectors. Nature. 2003:423:136-7.

29. Dabiré KR, Diabaté A, Namontougou M, Djogbenou L, Kengne P, Simard F, et al. Distribution of insensitive acetylcholinesterase (ace-1R) in Anopheles gambiae s.l. populations from Burkina Faso (West Africa). Trop Med Int Health. 2009:14:396-403.

30. Gorouhi MA, Vatandoost H, Oshaghi MA, Raeisi A, Enayati AA, Mirhendi $\mathrm{H}$, et al. Current susceptibility status of Anopheles stephensi (Diptera: Culicidae) to different imagicides in a malarious area, Southeastern of Iran. J Arthropod Borne Dis. 2016;10:493-500.

31. Safi NHZ, Ahmadi AA, Nahzat S, Warusavithana S, Safi N, Valadan R, et al. Status of insecticide resistance and its biochemical and molecular mechanisms in Anopheles stephensi (Diptera: Culicidae) from Afghanistan. Malar J. 2019;18:249.

32. Abbasi M, Hanafi-Bojd AA, Yaghoobi-Ershadi MR, Vatandoost H, Oshaghi MA, Hazratian T, et al. Resistance status of main malaria vector, Anopheles stephensi Liston (Diptera: Culicidae) to insecticides in a malaria Endemic Area, Southern Iran. Asian Pac J Trop Med. 2019;12:43-8.
33. Zare M, Soleimani-Ahmadi M, Davoodi SH, Sanei-Dehkordi A. Insecticide susceptibility of Anopheles stephensi to DDT and current insecticides in an elimination area in Iran. Parasit Vectors. 2016;9:571.

34. Ali Khan HA, Akram W, Lee S. Resistance to selected pyrethroid insecticides in the malaria mosquito, Anopheles stephensi (Diptera: Culicidae), From Punjab, Pakistan. J Med Entomol. 2018;55:735-8.

35. Ahmad M, Buhler C, Pignatelli P, Ranson H, Nahzat SM, Naseem M, et al. Status of insecticide resistance in high-risk malaria provinces in Afghanistan. Malar J. 2016;15:98.

36. Balkew M, Muntaser I, Koekemoer LL, Brooke BD, Engers H, Aseffa A, et al. Insecticide resistance in Anopheles arabiensis (Diptera: Culicidae) from villages in central, northern and south west Ethiopia and detection of kdr mutation. Parasit Vectors. 2010;3:40.

37. Yewhalaw D, Wassie F, Steurbaut W, Spanoghe P, Van Bortel W, Denis L, et al. Multiple insecticide resistance: an impediment to insecticide-based malaria vector control program. PLoS ONE. 2011;6:e16066.

38. Massebo F, Balkew M, Gebre-Michael T, Lindtjorn B. Blood meal origins and susceptibility of An arabiensis from Chano South-West Ethiopia. Parasit Vectors. 2013;6:44.

39. Asale A, Getachew Y, Hailesilassie W, Speybroeck N, Duchateau L, Yewhalaw D. Evaluation of the efficacy of DDT indoor residual spraying and longlasting insecticidal nets against insecticide resistant populations of Anopheles arabiensis Patton (Diptera: Culicidae) from Ethiopia using experimental huts. Parasit Vectors. 2014;7:131.

40. Messenger LA, Shililu J, Irish SR, et al. Insecticide resistance in Anopheles arabiensis from Ethiopia (2012-2016): a nationwide study for insecticide resistance monitoring. Malar J. 2017:16:469.

\section{Publisher's Note}

Springer Nature remains neutral with regard to jurisdictional claims in published maps and institutional affiliations.
Ready to submit your research? Choose BMC and benefit from:

- fast, convenient online submission

- thorough peer review by experienced researchers in your field

- rapid publication on acceptance

- support for research data, including large and complex data types

- gold Open Access which fosters wider collaboration and increased citations

- maximum visibility for your research: over $100 \mathrm{M}$ website views per year

At BMC, research is always in progress.

Learn more biomedcentral.com/submissions 